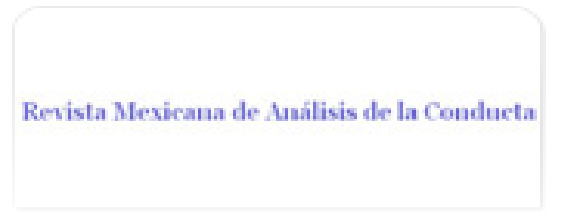

Revista Mexicana de Análisis de la Conducta ISSN: 0185-4534

editora@rmac-mx.org

Sociedad Mexicana de Análisis de la Conducta México

Ruiz, Jorge A.; Bruner, Carlos A.; Balderrama, Dulce M.

Efecto de tiempo relativo en demoras de reforzamiento señaladas y no señaladas

Revista Mexicana de Análisis de la Conducta, vol. 33, núm. 2, 2007, pp. 119-138

Sociedad Mexicana de Análisis de la Conducta

Guadalajara, México

Disponible en: http://www.redalyc.org/articulo.oa?id=59333202

- Cómo citar el artículo

- Número completo

- Más información del artículo

- Página de la revista en redalyc.org

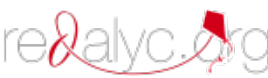

Sistema de Información Científica

Red de Revistas Científicas de América Latina, el Caribe, España y Portugal Proyecto académico sin fines de lucro, desarrollado bajo la iniciativa de acceso abierto 


\title{
EFECTO DE TIEMPO RELATIVO EN DEMORAS DE REFORZAMIENTO SEÑALADAS Y NO SEÑALADAS
}

\author{
RELATIVE TIME EFFECT IN SIGNALED AND UNSIGNALED \\ DELAYS OF REINFORCEMENT \\ JORGE A. RUIZ, CARLOS A. BRUNER \& DULCE M. BALDERRAMA ${ }^{1}$ \\ UNIVERSIDAD NACIONAL AUTÓNOMA DE MÉXICO
}

\begin{abstract}
RESUMEN
Un propósito del estudio fue determinar si correlacionando cada componente de 4 minutos de un programa múltiple con demoras de reforzamiento de 0,2 , 4 y 8 s se podría obtener un gradiente de demora de reforzamiento dentro de una misma sesión. En cada componente se entregó el reforzador conforme a un programa tándem compuesto de un intervalo al azar que sumado a cada demora nominal resultó en un intervalo entre reforzadores (IER) constante de $32 \mathrm{o}$ de $128 \mathrm{~s}$. Se asignaron tres palomas a cada IER. Las tasa de respuesta en cada componente del programa múltiple disminuyó gradualmente conforme se alargó la demora de reforzamiento. Un segundo propósito del estudio fue determinar el punto en un continuo definido por la probabilidad de señalar el periodo de demora ( $p(s e n ̃ a l))$ en el cual los efectos de frecuencia de reforzamiento se convierten en efectos de tiempo relativo en un procedimiento de demora de reforzamiento. La $p($ señal) fue de $0.00,0.33,0.66$ o 1.00. Cuando la $p$ (señal) fue de 0.00 . las tasas de respuesta ante cualquier demora fueron más altas con el IER de $32 \mathrm{~s}$ que con $128 \mathrm{~s}$, mostrando un efecto de frecuencia de reforzamiento. Cuando se incrementó el valor de $p$ (señal) de 0.33 a 1.00 las tasas de respuesta para cualquier demora fueron más altas con el IER de $128 \mathrm{~s}$ que con $32 \mathrm{~s}$, mostrando un efecto de tiempo relativo.
\end{abstract}

1. Dirigir correspondencia a: Laboratorio de Condicionamiento Operante, Facultad de Psicología, UNAM. Av. Universidad 3004, Cd. Universitaria. México, D.F. 04510. Email: cbruner@servidor. unam.mx oruizvja@yahoo.com. 
Palabras clave: Demora de reforzamiento, frecuencia de reforzamiento, tiempo relativo, palomas.

\begin{abstract}
One purpose of this study was to determine if by correlating each component of a multiple schedule with delays-of-reinforcement of either $0,2,4$, or $8 \mathrm{~s}$, a within-session delay-of-reinforcement gradient could be obtained. Reinforcement was delivered on each component using a tandem schedule composed by a random interval that added to each nominal delay yielded a constant interreinforcement interval (IRI) of either 32 or $128 \mathrm{~s}$. Three pigeons were assigned to each IRI. Response rates in each component of the multiple schedules decreased gradually as the reinforcement delay was lengthened. A second purpose was to determine the point on a continuum defined by the probability of signaling the delay period $p$ (signal) at which frequency-of-reinforcement effects become relative-time effects in a reinforcement-delay procedure. The $p$ (signal) was either $0.00,0.33,0,66$ or 1.00 . When $p$ (signal) was 0.00 , global response rates for any given delay were higher with the $32 \mathrm{~s}$ than with the $128 \mathrm{~s} \mathrm{IRI}$, showing a frequency-of-reinforcement effect. When $\mathrm{p}$ (signal) was increased from 0.33 to 1.00 global response rates for any given delay were higher with the $128 \mathrm{~s}$ than the $32 \mathrm{~s} \mathrm{IRI}$, showing a relative-time effect.

Key words: Delay of reinforcement, frequency of reinforcement, relative time, pigeons.
\end{abstract}

La tasa de una respuesta operante disminuye conforme se alarga el intervalo entre la respuesta y el reforzador (Deese \& Hulse, 1967; Kimble, 1961). A la disminución gradual en la tasa de respuesta con demoras de reforzamiento progresivamente más largas se le conoce como gradiente de demora (e.g., Sizemore \& Lattal, 1978). Este efecto aparece consistentemente en una diversidad de situaciones experimentales (cf. Renner, 1964; Schneider, 1990; Tarpy \& Sawabini, 1974).

En algunos estudios sobre demora de reforzamiento se han obtenido gradientes exponiendo a los mismos sujetos a demoras de diferente duración en bloques sucesivos de sesiones, incluso intercalando bloques de sesiones con reforzamiento inmediato entre dos duraciones de demora (e.g., Richards, 1981; Sizemore \& Lattal, 1978). En otros estudios se han obtenido gradientes de demora exponiendo a diferentes grupos de sujetos a diferentes duraciones de la demora (e.g., Bruner, Avila, Acuña \& Gallardo, 1998). Ambos diseños experimentales, intra-sujetos y entre-grupos, exigen un tiempo largo de exposición al procedimiento y en el caso de los diseños entre-grupos, un 
número grande de sujetos. Por esta razones, sería conveniente disponer de un método más eficiente para estudiar los efectos de la demora de reforzamiento. A la fecha sólo existe un intento de obtener gradientes de demora señalada y no señalada en una misma sesión experimental y con los mismos sujetos. Reilly y Lattal (2004) lograron replicar el conocido gradiente de demora con palomas utilizando un procedimiento en el que la duración de la demora aumentaba progresivamente en intervalos entre reforzadores sucesivos. En vista de la conveniencia de estudiar los efectos de la demora de reforzamiento con los mismos sujetos y en una misma sesión experimental, uno de los propósitos del presente estudio fue determinar si era posible establecer un gradiente de demora en una misma sesión utilizando un programa múltiple de reforzamiento en el que demoras de reforzamiento de diferente duración estaban asociadas con diferentes componentes.

Una variante de procedimiento para estudiar los efectos de la demora ha consistido en señalar con un estímulo neutral el intervalo respuesta-reforzador. En comparación con los resultados de estudios en los que no se señala el periodo de demora se ha encontrado que la tasa de respuesta ante cualquier demora de reforzamiento es más alta cuando se señala la demora (e.g., Azzi, Fix, Keller, \& Rocha e Silva, 1964; Richards, 1981; Schaal \& Branch, 1988). Este efecto se ha atribuido a la función de la señal como reforzador condicionado de la respuesta que inicia la demora (e.g., Grice, 1948; Spence, 1947, 1956).

En estudios sobre demora de reforzamiento no señalada, se ha encontrado que el resultado más común es que la tasa de respuesta bajo cualquier demora es más alta cuando el intervalo entre reforzadores es corto que cuando el intervalo entre reforzadores es largo (e.g., Bruner, Avila, Acuña \& Gallardo, 1998). Sin embargo, en procedimientos de demora señalada la tasa de respuesta bajo una misma demora es más alta cuando el intervalo entre reforzadores es largo que cuando es corto. Por ejemplo, Schaal, Schuh y Branch (1992) encontraron que demoras de reforzamiento señaladas de 5,10 y $20 \mathrm{~s}$ en combinación con un intervalo entre reforzadores de $20 \mathrm{~s}$ resultaron en tasas de respuesta más bajas que en combinación con un intervalo entre reforzadores de 120 s (véase también, Avila \& Bruner, 1989). Williams (1998) denominó a este efecto principio de tiempo relativo. El efecto de tiempo relativo es considerablemente robusto en diversas situaciones de reforzamiento señalado; por ejemplo, en estudios sobre la función de un estímulo como reforzador condicionado (e.g., Fantino, 1969) o como estímulo discriminativo (e.g., Schaal, Odum, Shahan, 2000; Terrace, 1966). De hecho, Williams concluyó que el principio de tiempo relativo solamente opera cuando existe una relación de contingencia entre una respuesta, un estímulo y un reforzador y no cuando solamente existe una contingencia entre la respuesta y el reforzador. 
Shahan y Lattal (2005) probaron la hipótesis de Williams (1998) replicando el estudio de Schaal, Schuh y Branch (1992) pero sin señalar las demoras de reforzamiento. Encontraron que alargar la demora de reforzamiento no señalada en combinación con un intervalo entre reforzadores largo resultó en una tasa de respuesta más baja que en combinación con un intervalo entre reforzadores corto. Shahan y Lattal concluyeron que sus resultados apoyaban la formulación de Williams. En conjunto, los estudios sobre demora de reforzamiento sugieren que si se señala la demora se obtiene un efecto de tiempo relativo y si no se señala la demora se obtiene un efecto de frecuencia de reforzamiento; esto es, una correlación positiva entre las tasas de respuesta y de reforzamiento.

En los procedimientos de reforzamiento demorado es posible considerar un continuo en el cual el caso en que la señal siempre precede al reforzador representa un extremo del continuo y el caso en que la señal nunca precede al reforzador representa el otro extremo del continuo. Una variable que permite explorar puntos intermedios de este continuo es la probabilidad de introducir la señal en el periodo de demora de reforzamiento. Lattal (1984) estudió el efecto de señalar intermitentemente una demora de reforzamiento constante de $4 \mathrm{~s}$ usando un intervalo entre reforzadores también constante de $60 \mathrm{~s}$. Usando palomas como sujetos, primero las expuso a una condición en que la señal nunca precedía al reforzador y en condiciones sucesivas introdujo la señal en 33, 66 y 100 \% de los intervalos de demora. Encontró que en cuatro de cinco palomas la tasa global de respuesta fue una función monotónica creciente de aumentar el porcentaje de demoras señaladas. Los hallazgos de Lattal sugieren que la probabilidad de presentar un estímulo durante la demora de reforzamiento puede ser una variable integradora de los efectos de frecuencia de reforzamiento y de tiempo relativo. Esto es, sugieren la pregunta relativa al punto en este continuo en el que por primera vez se observa un efecto o el otro y además, el punto en el que el efecto de frecuencia de reforzamiento se convierte en un efecto de tiempo relativo o viceversa.

\section{MÉTODO}

\section{Sujetos}

Se utilizaron 6 palomas mensajeras de 5 años de edad y experimentalmente ingenuas al inicio de la investigación. Se alojó a las palomas en jaulas individuales con acceso libre al agua y acceso restringido a la comida para mantenerlas al $80 \%$ de su peso en alimentación libre durante todo el experimento. 


\section{Aparatos}

Se utilizó una cámara experimental (BRS/LVE Mod. 20705) equipada con un ventilador que facilitó la circulación del aire dentro de la cámara. Se colocó una tecla (Med Associates Mod. ENV-130M) en el centro del panel frontal de la cámara a $22 \mathrm{~cm}$ respecto del piso, la cual requirió una fuerza de $0.25 \mathrm{~N}$ para que se registrara una respuesta y podía iluminarse de color rojo, verde, amarillo y blanco. Se activó un dispensador de comida (BRS/LVE Mod. GFM001) operado por un solenoide para permitir a los sujetos el acceso a una mezcla de granos durante $3 \mathrm{~s}$ a través de una apertura de $6 \times 6 \mathrm{~cm}$ ubicada debajo de la tecla central a $7 \mathrm{~cm}$ del piso de la caja. Un foco de 28 viluminó la cámara experimental durante cada una de las sesiones experimentales excepto en los periodos de acceso a la comida en los que únicamente un foco de 28 v iluminó el recipiente de la comida. En la parte posterior del panel frontal se instaló un generador de tonos (Sonalert SC628H 71036) que presentó un tono de $85 \mathrm{~dB}$ y una bocina (Quam 270-8110 3A05) que generó ruido blanco de $70 \mathrm{~dB}$ para enmascarar ruidos ajenos a la investigación. El control y registro de los eventos experimentales se realizó en un cuarto adyacente a donde se encontraba la cámara experimental.

\section{Procedimiento}

Todas las sesiones experimentales comenzaban con la iluminación de la cámara experimental y de la tecla. Las sesiones terminaban cuando cada sujeto obtenía 50 reforzadores o después de un tiempo predeterminado. Desde la primera sesión la tecla cambiaba a uno de cuatro colores cada cuatro minutos independientemente de la conducta de los sujetos. Los cambios de color ocurrieron al azar con la restricción de que no ocurriera el mismo color más de dos veces consecutivas.

\section{Establecimiento de la respuesta}

Para establecer el picoteo a la tecla se implementó un procedimiento de automoldeamiento consistente en un programa concurrente tiempo al azar (TA) $64 \mathrm{~s}$ reforzamiento continuo. Tres sesiones fueron suficientes para establecer la respuesta de picoteo en todos los sujetos. En la cuarta sesión se suspendió el programa de TA $64 \mathrm{~s}$. Independientemente de los colores en la tecla el programa de reforzamiento continuo para cada respuesta cambió a uno de intervalo al azar (IA) $16 \mathrm{~s}$ en los siguientes tres días. Con el objeto de generar persistencia de la respuesta el programa de IA 16 s se cambió a IA $32 \mathrm{~s}$ durante otras tres sesiones y posteriormente a IA 64 s durante otras cinco sesiones. Durante esta fase del estudio las sesiones duraban una hora o el tiempo necesario para obtener 50 reforzadores. Después de las catorce sesiones de entrenamiento a picar la tecla, se clasificó a los sujetos conforme a su tasa de respuesta y conforme a esta variable se les asignó a dos diferentes condicio- 
nes experimentales de tal forma que en cada condición hubiera una paloma con una tasa alta, otra con una tasa intermedia y otra con una tasa baja. La tasa de respuesta promedio de los dos grupos de palomas fue similar.

2. Programas múltiples de reforzamiento demorado con diferente intervalo entre reforzadores

Para los seis sujetos, a partir de la sesión 15 y hasta el final del experimento los cuatro colores de la tecla central se correlacionaron con una diferente demora de reforzamiento.

Para tres palomas el programa de reforzamiento vigente en cada color de la tecla fue un programa tándem IA t s TF 0, 2, 4 o $8 \mathrm{~s}$. Con cada cambio de color en la tecla se iniciaba el componente de IA cuyo valor fue el complemento del TF de tal forma que la duración conjunta de ambos componentes fuera de $32 \mathrm{~s}$. Para las otras tres palomas el programa de reforzamiento vigente en cada color de la tecla fue un programa tándem IA t s TF 0, 2, 4 o $8 \mathrm{~s}$. Con cada cambio de color en la tecla se iniciaba el componente de IA cuyo valor fue el complemento del TF de tal forma que la duración conjunta de ambos componentes fuera de $128 \mathrm{~s}$. Estas condiciones experimentales se mantuvieron durante 60 sesiones.

3. La variación en la probabilidad de una señal durante la demora

En la condición precedente nunca se señalaron las diferentes demoras de reforzamiento (i.e., $p($ señal) 0.00). Durante las siguientes 30 sesiones experimentales se programó la ocurrencia de un tono durante el periodo de demora de reforzamiento conforme a una probabilidad de ocurrencia de 0.33. Durante las siguientes 30 sesiones la p(señal) cambió a 0.66 y durante otras 30 sesiones cambió a 1.00. Durante esta última condición murió una paloma (Sujeto 1) asignada al programa múltiple de 32 s como intervalo entre reforzadores. Para todas las condiciones con una diferente $p$ (señal) las sesiones experimentales terminaban después de que cada sujeto obtuvo 50 reforzadores o después de 30 minutos para la condición del intervalo entre reforzadores (IER) de 32 s o 120 minutos para la condición del IER de 128 s.

Las duraciones de la demora de reforzamiento, del intervalo entre reforzadores, así como las probabilidades de presentar la señal durante el periodo de demora que se emplearon en el estudio se eligieron con base en que en estudios anteriores se ha encontrado que valores semejantes son conducentes a un efecto robusto sobre la tasa de respuesta (cf., Bruner, Avila, Acuña \& Gallardo, 1998; Lattal, 1984; Schaal, Schuh \& Branch, 1992). Se utilizó un tono de $85 \mathrm{~dB}$ debido a que se ha mostrado que es una intensidad audible en palomas (e.g., Nakajima, 1994). El criterio para decidir el número de sesiones bajo cada valor de $\mathrm{p}$ (señal) fue arbitrario, pero razonable para permitir que la tasa de respuesta se estabilizara en cada condición. 


\section{RESULTADOS}

En la descripción de los datos obtenidos en este estudio se prefirió presentar las medias de los tres sujetos asignados a un diferente intervalo entre reforzadores por las siguientes razones: Primero, porque los datos sumarios se prestan con mayor facilidad para describir los efectos de las combinaciones de las variables independientes. Segundo, porque el haber igualado a las palomas en las dos condiciones conforme a su tasa de picoteo pretendió minimizar las diferencias individuales y por lo tanto establecer la representatividad de las funciones promedio. Tercero, porque es común emplear promedios al describir fenómenos de demora de reforzamiento debido a que en algunas ocasiones la variación en los datos individuales tienden a distraer la atención de los efectos molares. Cuarto, porque presentar datos individuales hubiera implicado un mayor número de figuras a las que se presentan en esta sección. Con el propósito de cuantificar la concordancia entre los datos individuales y la media de ellos para la tasa global de respuesta, la tasa de respuesta durante el IA y para la tasa de respuesta durante el periodo de demora de reforzamiento se calcularon coeficientes de concordancia de Kendall (cf. Daniel, 1978). Se encontró que todos los valores del coeficiente de concordancia entre la media y los datos individuales tanto de los sujetos bajo el intervalo entre reforzadores de $32 \mathrm{~s}$ como de los sujetos bajo el intervalo de $128 \mathrm{~s}$ fueron significativos con una probabilidad menor a 0.01 , con valores de $m=16$ y $n=4$ (tasa global: $\underline{\mathrm{W}}=0.44$ y 0.33 , respectivamente; tasa durante el IA: $\underline{W}=0.48$ y 0.37 , respectivamente y; tasa durante la demora: $\underline{W}=0.26$ y 0.38 respectivamente). Estos coeficientes muestran que las funciones promedio representan confiablemente a las funciones individuales.

En la Figura 1 se muestra la tasa global media de los tres sujetos en cada intervalo entre reforzadores en función de la demora nominal de reforzamiento y de la probabilidad de la señal en los periodos de demora. La razón para presentar este análisis es que la tasa global de respuesta es la variable dependiente más común en diferentes estudios sobre demora de reforzamiento. La gráfica de la izquierda muestra este dato para el intervalo entre reforzadores de $32 \mathrm{~s}$ y la de la derecha para el de $128 \mathrm{~s}$. Estas medias se obtuvieron promediando las últimas cinco sesiones en cada combinación de las variables independientes. Para ambos intervalos entre reforzadores, 32 y $128 \mathrm{~s}$, la tasa global de respuesta fue una función decreciente de alargar la demora nominal de reforzamiento y una función creciente de aumentar la probabilidad de presentar una señal durante el periodo de demora. Para p(señal) 0.00 en combinación con reforzamiento inmediato la tasa global media fue más alta cuando el intervalo entre reforzadores fue de $32 \mathrm{~s}$ que cuando fue de $128 \mathrm{~s}$. Sin embargo, las funciones obtenidas con $p($ señal $)=$ 
0.33, 0.66 y 1.00 en combinación con demoras de 2,4 y $8 \mathrm{~s}$ fueron más altas cuando el intervalo entre reforzadores fue de 128 que cuando fue de $32 \mathrm{~s}$.
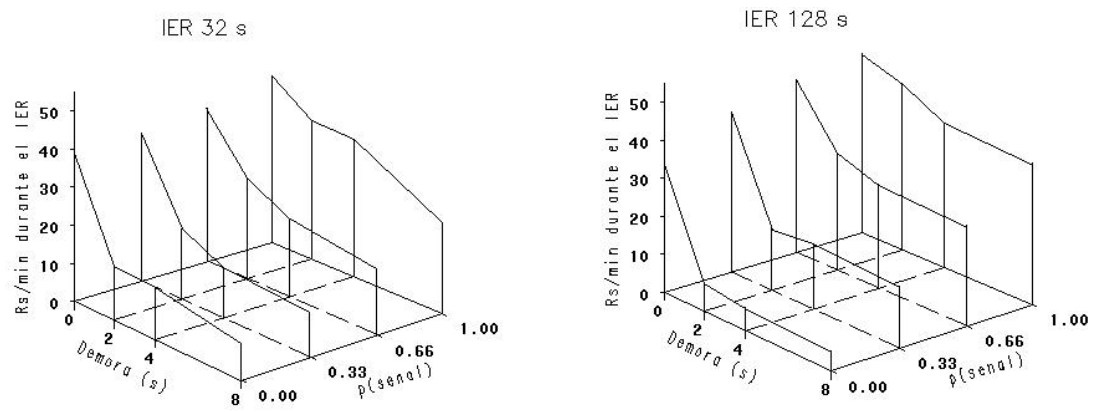

Figura 1. Tasa global de respuesta en función de la duración de la demora de reforzamiento y del valor de $p$ (señal), bajo las dos duraciones del intervalo entre reforzadores (IER).

En algunos estudios sobre demora de reforzamiento se reportan por separado las tasas medias de respuesta durante cada componente del programa tándem de demora de reforzamiento, por lo que fue importante observar la tasa de respuesta por separado durante el IA y durante la demora de reforzamiento en el presente experimento (e.g., Schaal \& Branch, 1988; Shahan \& Lattal, 2005). En la Figura 2 se muestra la tasa media durante el componente previo a la demora de reforzamiento. Este dato se presenta en el mismo formato que la figura anterior. La tasa media durante el primer componente del programa tándem (IA t s) disminuyó gradualmente conforme se alargó la demora de reforzamiento y aumentó gradualmente conforme se aumentó $\mathrm{p}$ (señal) bajo ambas duraciones del intervalo entre reforzadores. Con $\mathrm{p}$ (señal) 0.00 la tasa media para la función de demora obtenida con el intervalo entre reforzadores de $32 \mathrm{~s}$ fue más alta que con el intervalo de $128 \mathrm{~s}$. Sin embargo, cuando $p$ (señal) fue $0.33,0.66$ y 1.00 la tasa de respuesta media en el primer componente del programa tándem fue más alta ante cualquier demora cuando el intervalo entre reforzadores fue de $128 \mathrm{~s}$ que cuando fue de $32 \mathrm{~s}$.

En la Figura 3 se muestra la tasa media de respuesta durante el componente de demora del programa tándem (TF 0, 2, 4 y $8 \mathrm{~s}$ ). Sin considerar la condición de reforzamiento inmediato los datos se presentan en el mismo formato de las figuras anteriores. Se encontró que el efecto de la demora fue diferente dependiendo de la $p$ (señal) y el intervalo entre reforzadores. Para ambos intervalos entre reforzadores, la tasa media no varió sistemáticamente 
IER $32 \mathrm{~s}$

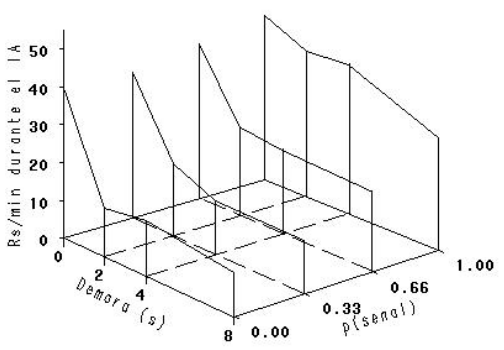

IER $128 \mathrm{~s}$

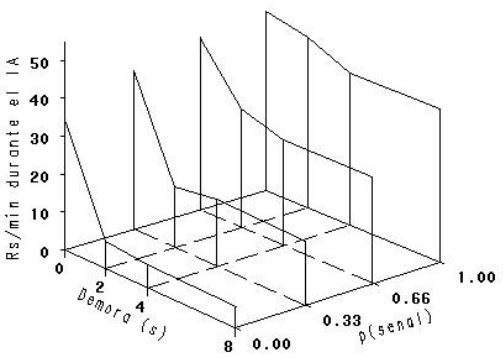

Figura 2. Tasa de respuesta durante el intervalo al azar en función de la duración de la demora de reforzamiento y del valor de $p$ (señal), bajo las dos duraciones del intervalo entre reforzadores (IER).

con la demora nominal de reforzamiento con $p$ (señal) 0.00 , pero fue más alta con el intervalo entre reforzadores de 32 que con el de $128 \mathrm{~s}$. Con p(señal) 0.33, 0.66 y 1.00 en combinación con el intervalo entre reforzadores de 32 $\mathrm{s}$, la tasa media disminuyó conforme se alargó la demora. Sin embargo, las mismas $p$ (señal) en combinación con el intervalo entre reforzadores de $128 \mathrm{~s}$, controlaron tasas comparativamente más planas ante las diferentes demoras de reforzamiento. Si acaso, las tasas medias disminuyeron ligeramente con la demora de $8 \mathrm{~s}$. Para todas las demoras de reforzamiento la tasa media de respuesta durante la demora fue más alta con el intervalo entre reforzadores de $128 \mathrm{~s}$ que el intervalo de $32 \mathrm{~s}$.

IER $32 \mathrm{~s}$

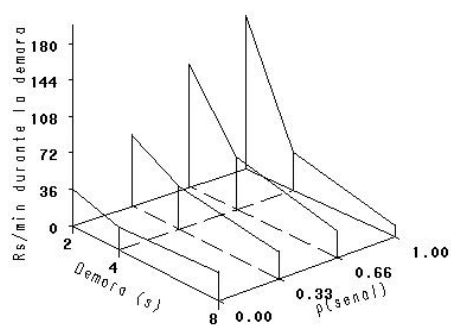

IER $128 \mathrm{~s}$

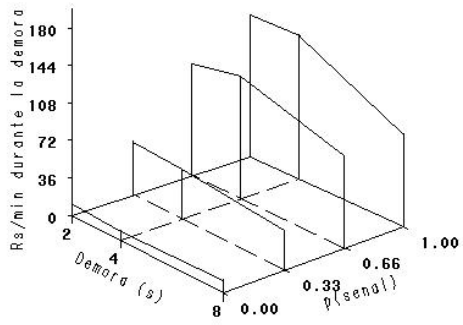

Figura 3. Tasa de respuesta durante el periodo de demora de reforzamiento en función de la duración de la demora de reforzamiento y del valor de $p$ (señal), bajo las dos duraciones del intervalo entre reforzadores (IER). 
Cuando $p$ (señal) fue 0.00 nunca se señaló el periodo de demora. Cuando $p$ (señal) fue 1.00 siempre se señaló el periodo de demora. Cuando $p$ (señal) fue 0.33 y 0.66 el periodo de demora de reforzamiento se señaló intermitentemente, por lo que resultó importante determinar si las tasas medias de respuesta en presencia de la señal fueron semejantes a las que ocurrieron en ausencia de la señal. En la Figura 4 se muestra la tasa media de respuesta durante el periodo de demora en aquellas ocasiones en las que se presentó la señal y en las que no se presentó la señal. Los paneles superiores muestran la tasa promedio con $p$ (señal) 0.33 y los inferiores muestran la tasa promedio con $\mathrm{p}$ (señal) 0.66 . Los paneles de la izquierda muestran la misma variable dependiente cuando se presentó la señal y los de la derecha cuando no se presentó la señal. En cada panel se presenta por separado la tasa promedio de los sujetos con el intervalo entre reforzadores de 32 y de $128 \mathrm{~s}$. Se encontró que cuando $\mathrm{p}$ (señal) fue 0.33 y 0.66 la tasa media fue una función decreciente de alargar la demora de reforzamiento, pero solo cuando se presentó la señal dado que en su ausencia la tasa media no varió sistemáticamente con la demora. La tasa media durante la señal fue más alta con el intervalo entre reforzadores de $128 \mathrm{~s}$ que con el intervalo de $32 \mathrm{~s}$ y el efecto fue más pronunciado con $p$ (señal) 0.66 que con 0.33 . La tasa media en ausencia de la señal no varió con el intervalo entre reforzadores.

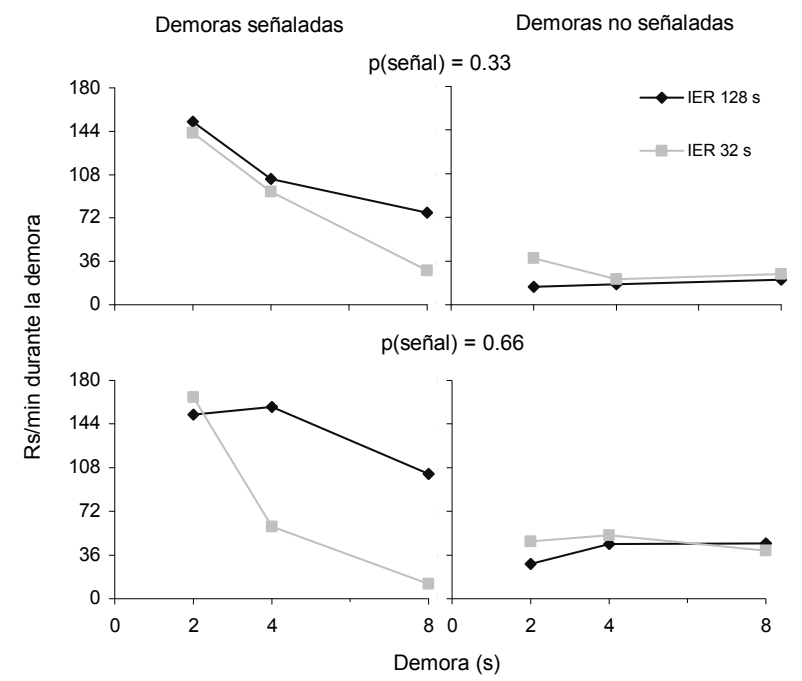

Figura 4. Tasa de respuesta durante los periodos de demora de reforzamiento señalados y no señalados en las condiciones de $p$ (señal) $=0.33 y$ 0.66 , bajo las dos duraciones del intervalo entre reforzadores (IER). 
Los datos de la Figura 4 sugieren un incremento global en la tasa de respuesta durante los periodos de demora de reforzamiento al aumentar el valor de $p$ (señal) de 0.33 a 0.66 . Esto es, que la frecuencia con la que se presentó la señal durante los periodos de demora tuvo un efecto graduado sobre la tasa de respuesta durante estos periodos. Ante la posibilidad de que los efectos de presentar la señal durante la demora hayan sido de todo o nada, se efectuó un análisis de varianza para un diseño mixto $2 \times 2 \times 2 \times 3$ (2 intervalos entre reforzadores: 32 y $128 \mathrm{~s}$; presencia vs. ausencia de la señal; 2 valores de $p$ (señal): 0.33 y 0.66 y; 3 demoras: 2,4 y 8 s). El ANOVA mostró que sólo el efecto principal de la demora y su interacción con la presencia vs. ausencia de la señal, así como la interacción de los cuatros factores considerados en el modelo fueron significativos con una probabilidad menor a $0.05(\underline{E}(2,8)=$ 13.79, $\underline{F}(2,8)=16.583, \underline{F}(2,8)=5.566$, respectivamente). Para determinar el efecto de la interacción entre el intervalo entre reforzadores, la presencia vs. ausencia de la señal, el valor de p(señal) y la duración de la demora de reforzamiento se realizó por separado un análisis de varianza de medidas repetidas con tres factores (presencia vs. ausencia de la señal, valor de $p$ (señal) y duración de la demora) para las palomas expuestas a los dos intervalos entre reforzadores de diferente duración. Para las palomas bajo el intervalo entre reforzadores de $32 \mathrm{~s}$ se encontró que sólo el efecto principal de la demora y su interacción con la presencia vs. ausencia de la señal fueron significativos con una probabilidad menor a $0.05(\underline{F}(2,4)=9.537$ y $\underline{F}(2,4)=7.62$, respectivamente). En cuanto a las palomas bajo el intervalo entre reforzadores de 128 s se encontró que sólo la interacción entre la duración de la demora y la presencia vs. ausencia de la señal fue significativa con una probabilidad menor a $0.05(\underline{E}(2,4)=16.695)$. Es posible concluir que el aumento global en la tasa de respuesta durante los periodos de demora de reforzamiento se debió al incremento en la probabilidad de presentar la señal aunque este efecto no fue lo suficientemente robusto para alcanzar significancia estadística.

En estudios sobre reforzamiento demorado es posible que conforme se alarga la demora concomitantemente se disminuya la frecuencia de reforzamiento (cf. Lattal, 1987). En el presente estudio se determinó si la frecuencia de reforzamiento pudo haber controlado la tasa de respuesta a través de las diferentes condiciones experimentales. En la Tabla 1 se muestra para cada sujeto la tasa media de reforzamiento durante las últimas cinco sesiones de cada condición en función de las variables independientes del estudio. Un análisis de varianza para un diseño mixto $2 \times 4 \times 4$ (2 intervalos entre reforzadores: 32 y 128 s; 4 demoras: 0, 2, 4 y 8 s y; 4 valores de p(señal): 0.00 , $0.33,0.66$ y 1.00) mostró que sólo los efectos principales del intervalo entre reforzadores, de la demora y de la probabilidad de señalar las demoras, así como la interacción entre el intervalo entre reforzadores y la duración de la demora, fueron significativos con una probabilidad menor a $0.05(\underline{F}(1,3)=$ 
41.672, $\underline{F}(3,9)=40.391, \underline{F}(3,9)=16.145, \underline{F}(3,9)=17.77$, respectivamente). Por medio de pruebas de contraste se determinó que sólo las diferencias entre $p($ señal $)=0.33$ y 1.00 en relación a 0.00 , así como entre 0.33 y 1.00 , fueron significativas con una probabilidad menor a $0.05(\underline{F}(1,3)=11.513, \underline{F}(1,3)$ $=114.786, \underline{F}(1,3)=40.221$, respectivamente). Para determinar el efecto de la interacción entre el intervalo entre reforzadores y la duración de la demora de reforzamiento se realizó por separado un análisis de varianza de medidas repetidas con un solo factor (4 duraciones de la demora) para las palomas expuestas a los dos intervalos entre reforzadores de diferente duración. Por medio de pruebas de contraste se encontró que para los sujetos bajo el intervalo entre reforzadores de $32 \mathrm{~s}$ sólo la diferencia entre las demoras de 4 y $8 \mathrm{~s}$ fue significativa con una probabilidad menor a $0.05(\underline{F}(1,1)=213.160)$. En cuanto a las palomas bajo el intervalo de $128 \mathrm{~s}$, se encontró que sólo las diferencias entre las demoras de 2,4 y $8 \mathrm{~s}$ en relación a $0 \mathrm{~s}$ fueron significativas con una probabilidad menor a $0.05(\underline{F}(1,1)=71.063, \underline{F}(1,1)=31.125$, $\underline{F}(1,1)=407.579$, respectivamente). De acuerdo a los resultados arrojados por el análisis de varianza es posible concluir que aunque se encontraron algunas diferencias significativas en la tasa de reforzamiento, éstas siguieron un patrón asistemático entre las diferentes combinaciones de las variables independientes del estudio. Por lo tanto, es probable que los efectos ordenados en las tasa global de respuesta, en la tasa durante el IA y durante la demora se hayan debido a la manipulación sistemática de las variables independientes más que a un efecto de una tasa de reforzamiento diferente en cada condición.

\begin{tabular}{|c|c|c|c|c|c|c|c|c|c|c|c|c|}
\hline \multirow[b]{4}{*}{$\mathrm{p}$ (señal) } & \multicolumn{12}{|c|}{ Duración promedio del intervalo entre reforzadores } \\
\hline & \multicolumn{6}{|c|}{$32 \mathrm{~s}$} & \multicolumn{6}{|c|}{$128 \mathrm{~s}$} \\
\hline & \multirow[b]{2}{*}{ Sujeto } & \multicolumn{4}{|c|}{ Duración de la demora } & \multirow[b]{2}{*}{ Sesiones } & \multirow[b]{2}{*}{ Sujeto } & \multicolumn{4}{|c|}{ Duración de la demora } & \multirow[b]{2}{*}{ Sesiones } \\
\hline & & 0 & 2 & 4 & 8 & & & 0 & 2 & 4 & 8 & \\
\hline & 1 & 1.27 & 1.20 & 1.07 & 0.81 & 60 & 4 & 0.14 & 0.09 & 0.12 & 0.07 & 60 \\
\hline \multirow[t]{2}{*}{0.00} & 2 & 1.27 & 0.81 & 0.92 & 0.71 & 60 & 5 & 0.33 & 0.17 & 0.19 & 0.21 & 60 \\
\hline & 3 & 1.20 & 1.04 & 1.25 & 1.23 & 60 & 6 & 0.40 & 0.27 & 0.20 & 0.15 & 60 \\
\hline \multirow{3}{*}{0.33} & 1 & 1.14 & 1.17 & 1.39 & 1.28 & 30 & 4 & 0.36 & 0.23 & 0.27 & 0.19 & 30 \\
\hline & 2 & 1.23 & 0.96 & 0.54 & 1.06 & 30 & 5 & 0.45 & 0.29 & 0.27 & 0.33 & 30 \\
\hline & 3 & 1.86 & 1.26 & $\begin{array}{l}1.22 \\
\end{array}$ & 1.08 & 30 & 6 & 0.36 & 0.29 & 0.32 & 0.40 & 30 \\
\hline \multirow{3}{*}{0.66} & 1 & 1.26 & 0.96 & 1.20 & 0.87 & $5^{*}$ & 4 & 0.40 & 0.20 & 0.21 & 0.24 & 30 \\
\hline & 2 & 1.29 & 1.05 & 0.70 & 0.86 & 30 & 5 & 0.44 & 0.43 & 0.40 & 0.41 & 30 \\
\hline & 3 & 1.71 & 1.75 & 1.51 & 1.34 & 30 & 6 & 0.39 & 0.43 & 0.34 & 0.36 & 30 \\
\hline \multirow{3}{*}{1.00} & & & & & & & 4 & 0.33 & 0.39 & 0.40 & 0.46 & 30 \\
\hline & 2 & 1.83 & 1.29 & 1.34 & 1.14 & 30 & 5 & 0.44 & 0.39 & 0.36 & 0.42 & 30 \\
\hline & 3 & 1.87 & 1.62 & 1.45 & 1.63 & 30 & 6 & 0.45 & 0.36 & 0.35 & 0.37 & 30 \\
\hline
\end{tabular}

Tabla 1. Tasa de reforzamiento para cada sujeto en función de la duración de la demora de reforzamiento, del intervalo entre reforzadores y de la probabilidad de señalar el periodo de demora. 
En los estudios sobre demora de reforzamiento en los que las respuestas que ocurren después de la respuesta que inicia la demora no tienen ninguna consecuencia, es común que las demoras obtenidas sean de menor duración que las programadas (e.g., Bruner, Avila, Acuña \& Gallardo, 1998). En la Figura 5 se muestra la duración obtenida de la demora de reforzamiento en términos de la media de los tres sujetos en cada intervalo entre reforzadores en función de la demora nominal de reforzamiento. Estas medias se obtuvieron durante las últimas cinco sesiones en cada combinación de las variables independientes. Se encontró que bajo cualquier combinación de duraciones de la demora, del intervalo entre reforzadores y de los valores de la probabilidad de señalar la demora de reforzamiento, la duración obtenida de la demora fue una función creciente de alargar la duración nominal de la demora de reforzamiento. Con $p$ (señal) 0.00 se encontró que la duración obtenida de la demora de reforzamiento fue mayor bajo el intervalo entre reforzadores de $128 \mathrm{~s}$ que bajo el intervalo de $32 \mathrm{~s}$. Sin embargo, conforme aumentó el valor de p(señal) de 0.33 a 1.00 , la duración obtenida de la demora de reforzamiento fue progresivamente menor con el intervalo entre reforzadores de $128 \mathrm{~s}$ que con el intervalo de $32 \mathrm{~s}$. Conforme a las variaciones ordenadas de la duración obtenida de las demoras de reforzamiento es posible concluir que la proximidad temporal entre la respuesta y el reforzador controló a la tasa de respuesta de manera más sistemática que la frecuencia obtenida de reforzamiento.

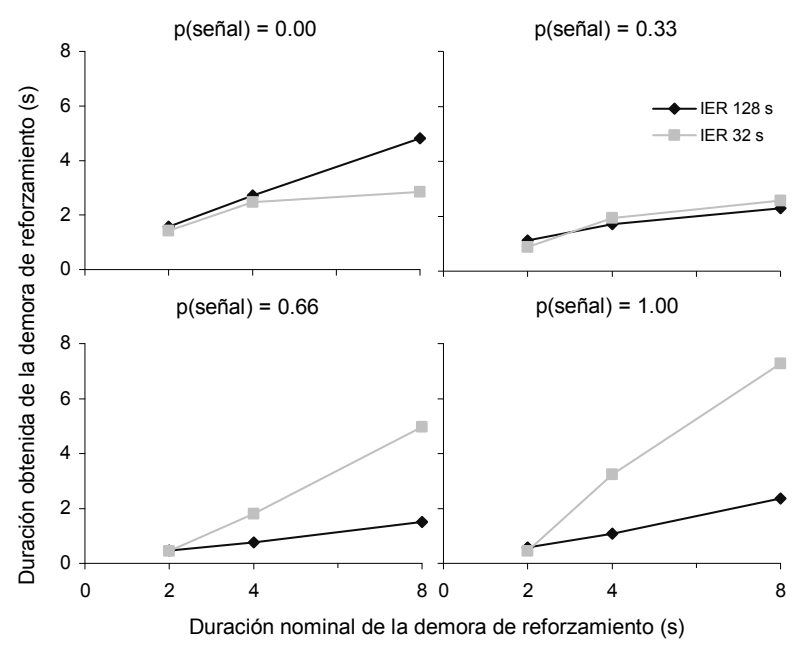

Figura 5. Duración obtenida de la demora de reforzamiento en función de la duración nominal de la demora de reforzamiento y del valor de $p$ (señal), bajo las dos duraciones del intervalo entre reforzadores (IER). 
Con el mismo formato que la Figura 4, en la Figura 6 se muestra la media de la duración obtenida de la demora de reforzamiento, en aquellas ocasiones en las que se presentó la señal y en las que no se presentó la señal en las condiciones en las que $p$ (señal) fue 0.33 y 0.66 . Se encontró que tanto en las demoras en las que se presentó la señal como en las que no, la duración obtenida de la demora de reforzamiento fue una función creciente de la duración nominal de la demora de reforzamiento. Se encontró que con p(señal) 0.33 la duración obtenida de la demora durante las demoras señaladas fue mayor con el intervalo entre reforzadores de $128 \mathrm{~s}$ que con el intervalo de 32 $\mathrm{s}$, pero con $\mathrm{p}$ (señal) 0.66 esta relación se invirtió. Durante las demoras no señaladas se encontró que bajo ambos valores de $p$ (señal), 0.33 y 0.66 , las duraciones obtenidas de la demora fueron muy similares entre los sujetos expuestos a las dos duraciones del intervalo entre reforzadores.

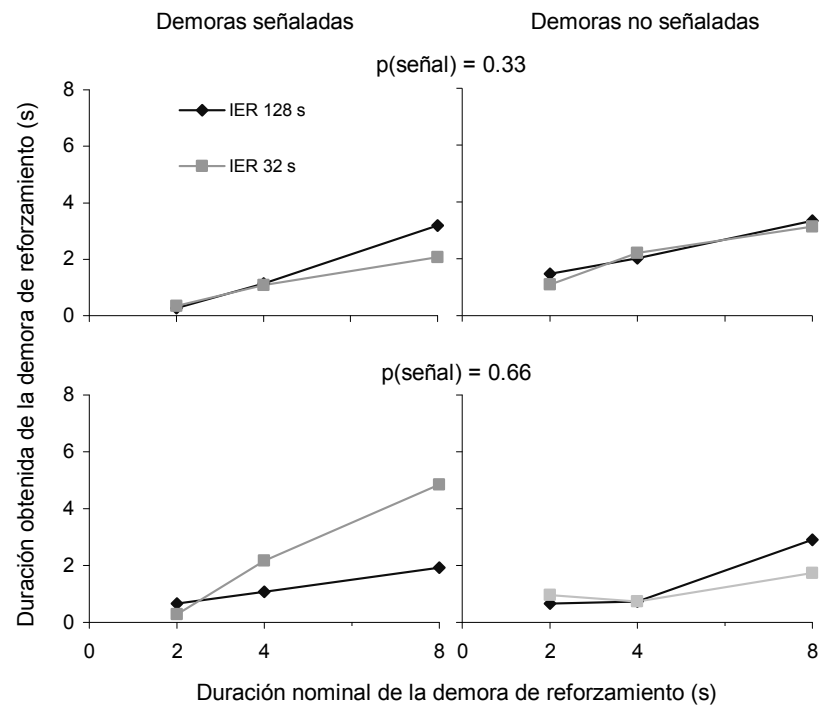

Figura 6. Duración obtenida de la demora de reforzamiento en función de la duración nominal de la demora durante los periodos de demora de reforzamiento señalados y no señalados en las condiciones de $p($ señal) $=0.33$ y 0.66 , bajo las dos duraciones del intervalo entre reforzadores (IER). 


\section{DISCUSIÓN}

El presente experimento se condujo por dos razones. La primera fue probar la utilidad de un programa múltiple de reforzamiento para obtener gradientes de demora de reforzamiento con los mismos sujetos y durante una misma sesión experimental. La segunda razón fue determinar el punto en un continuo definido por la probabilidad de presentar una señal durante la demora de reforzamiento en el que el efecto de frecuencia de reforzamiento se convierte en un efecto de tiempo relativo y viceversa.

Respecto a la posibilidad de obtener un gradiente de demora en una misma sesión usando un programa múltiple de reforzamiento, se encontró que tanto la tasa global de respuesta como la tasa de respuesta durante el IA fueron una función decreciente de alargar la demora de reforzamiento. Este efecto se replicó bajo las dos duraciones del intervalo entre reforzadores y bajo los cuatro valores de $p$ (señal). Dado que es común que en estudios sobre demora de reforzamiento se confundan los efectos de las demoras nominales con diferentes frecuencias y demoras obtenidas de reforzamiento, en el presente estudio se determinó si los resultados pudieron deberse a cualquiera de estas variables extrañas (cf., Sizemore \& Lattal, 1978; Schaal $\&$ Branch, 1988). Aunque un análisis de varianza mostró algunas diferencias significativas en la tasa de reforzamiento, la mayoría de estas fueron sólo entre las duraciones de demora para los sujetos bajo el intervalo entre reforzadores de $128 \mathrm{~s}$. De hecho, sólo las duraciones de 2, 4 y $8 \mathrm{~s}$ resultaron en una tasa de reforzamiento diferente comparada con la demora de $0 \mathrm{~s}$ para los sujetos bajo el intervalo entre reforzadores de $128 \mathrm{~s}$; mientras que bajo el intervalo de $32 \mathrm{~s}$, solo las demoras de 4 y $8 \mathrm{~s}$ difirieron entre si. Si bien la frecuencia de reforzamiento varió de manera no sistemática en función de las combinaciones de las variables independientes del estudio, se encontró que las demoras de reforzamiento obtenidas bajo cualquier combinación de demoras nominales con un diferente intervalo entre reforzadores y con los valores de $\mathrm{p}$ (señal) fueron consistentemente más cortas que las programadas, pero invariablemente siguieron el mismo orden de magnitud. Por lo tanto, de las dos variables que pudieron confundirse con los efectos de la demora nominal de reforzamiento sólo la demora obtenida pudo haber afectado los resultados, pero solo en la misma dirección que la hipótesis original.

Reilly y Lattal (2004) reportaron que manteniendo constante un programa de intervalo variable (IV) $60 \mathrm{~s}$ y aumentando progresivamente la duración de una demora de reforzamiento de un intervalo entre reforzadores al siguiente en pasos de $2 \mathrm{~s}$, fue posible establecer un gradiente de demora en una misma sesión, utilizando incluso duraciones de la demora de hasta $160 \mathrm{~s}$. Los hallazgos de Reilly y Lattal son importantes para la literatura sobre demora de reforzamiento porque mostraron que es posible medir en una 
misma sesión el efecto de diferentes demoras de reforzamiento sobre la tasa de respuesta. También mostraron que no es necesario emplear un número grande de sujetos, de grupos y de condiciones experimentales para realizar un experimento en el que se estudien los valores de diferentes variables que modulan el efecto de la demora sobre la tasa de respuesta. La razón para no utilizar el mismo método en el presente estudio fue que variar la demora programada en cada intervalo entre reforzadores de la misma sesión habría dificultado la exploración paramétrica del efecto de variar la probabilidad de señalar los periodos de demora de reforzamiento. Por esta razón en este trabajo se prefirió correlacionar una diferente demora de reforzamiento fija con cada componente de un programa múltiple de reforzamiento. De acuerdo con los resultados obtenidos es posible concluir que el uso de un programa múltiple de reforzamiento es conducente al establecimiento de gradientes de demora de reforzamiento en una misma sesión (véase Lattal, 1987; Renner, 1964; Schneider, 1990; Tarpy \& Sawabini, 1974; como revisiones del efecto de la demora de reforzamiento y de la generalidad del gradiente de demora de reforzamiento en diversos procedimientos).

El segundo propósito del estudio consistió en averiguar el punto en el continuo definido por diferentes valores de $p$ (señal) en el cual los efectos de la demora de reforzamiento en combinación con intervalos entre reforzadores cortos o largos se comportan conforme al principio de frecuencia de reforzamiento o de tiempo relativo. Como se esperaba, en los extremos del continuo cuando nunca se señalaron las demoras ( $p($ señal) 0.00 ) y cuando siempre se señalaron las demoras ( $p$ (señal) 1.00), se duplicaron efectos conocidos. Cuando no se señalaron las demoras ( $p($ señal) 0.00 ) se encontró que ante las mismas demoras nominales de reforzamiento la tasa de respuesta global, durante el componente de IA y durante la demora fueron más altas con el intervalo entre reforzadores de $32 \mathrm{~s}$ que con el intervalo de $128 \mathrm{~s}$; un efecto semejante al de disminuir la frecuencia de reforzamiento. Estos resultados son consistentes con los de Bruner, Avila, Acuña y Gallardo (1998) quienes encontraron que ante demoras no señaladas en el rango de 0 a $24 \mathrm{~s}$ combinadas con tasas de reforzamiento altas (e,g., IA $15 \mathrm{~s}$ ) la tasa de respuesta era más alta que con tasas de reforzamiento bajas (e.g., IA 120 s). También son consistentes con los resultados de Shahan y Lattal (2005) quienes encontraron que aumentar la duración de una demora de reforzamiento no señalada de 0.5 a $8 \mathrm{~s}$ en combinación con dos programas de reforzamiento IV 20 y 120 s, resultó en tasas de respuesta más altas para el intervalo entre reforzadores corto que para el largo. En contraste con estos resultados, cuando se señalaron todas las demoras ( $p$ (señal) 1.00), las tasas de respuesta globales, durante el componente de IA y durante la demora fueron más altas con el intervalo entre reforzadores de $128 \mathrm{~s}$ que con el intervalo de $32 \mathrm{~s}$. Esto es, se encontró un efecto de tiempo relativo. Estos resultados son consisten- 
tes con los reportados en la literatura. Por ejemplo, Schaal, Schuh y Branch (1992) reportaron que duraciones de la demora de reforzamiento señalada de 5,10 y 20 s en combinación con intervalos entre reforzadores de 20 y 120 $s$ resultaron en tasas de respuesta más altas en el componente anterior a la demora con el intervalo entre reforzadores largo que con el intervalo corto.

Variar la probabilidad de presentar una señal durante la demora en los grados intermedios de p(señal) 0.33 y 0.66 resultó en que ante todas las demoras la tasa de respuesta global, antes y durante la señal, aumentó concomitantemente con las dos duraciones del intervalo entre reforzadores. Estos resultados son consistentes con los de Lattal (1984), quien sólo utilizó una duración del intervalo entre reforzadores y de la demora de reforzamiento. Lattal encontró que incrementar el porcentaje de demoras de reforzamiento señaladas resultó en un incremento en la tasa de respuesta. Además, dado que en el presente estudio se manipularon de forma paramétrica la duración del intervalo entre reforzadores y de la duración de la demora de reforzamiento, fue posible determinar que cuando la probabilidad de señalar los periodos de demora de reforzamiento fue $p$ (señal) 0.00 ocurrió un efecto semejante al de frecuencia de reforzamiento y que cuando la probabilidad de presentar la señal fue $p$ (señal) 1.00 ocurrió un efecto de tiempo relativo. Con $p$ (señal) de 0.33 y 0.66 se encontró una transición aparentemente gradual entre un efecto de frecuencia de reforzamiento con $p$ (señal) 0.00 , a un efecto de tiempo relativo con p(señal) 1.00 . En el presente estudio se encontró que basta con señalar los periodos de demora de reforzamiento con una probabilidad $p($ señal $)=0.33$ o tal vez menor para observar cambios ordenados en la tasa global de respuesta dirigidos hacia un efecto de tiempo relativo

Aunque los resultados de variar la $p$ (señal) fueron consistentes con los reportados en literatura, si se observa detalladamente la tasa de respuesta durante el IA y durante el periodo de demora en aquellas ocasiones en las que se presentó la señal y en las que no se presentó la señal se encuentran indicios que sugieren que los efectos de frecuencia de reforzamiento y de tiempo relativo son fenómenos cualitativamente diferentes, como lo han sugerido algunos autores (e.g., Shahan \& Lattal, 2005; Williams, 1998, 2003). Una contribución del presente estudio es el análisis de la tasa de respuesta durante las demoras de reforzamiento señaladas y no señaladas cuando $p$ (señal) fue 0.33 y 0.66 . Se encontró que la tasa de respuesta durante las demoras señaladas fue una función decreciente de alargar la duración de la demora, siendo más pronunciada la disminución en las tasas de respuesta con el intervalo entre reforzadores de $32 \mathrm{~s}$ que con el intervalo de $128 \mathrm{~s}$. Esta diferencia fue mayor con $p$ (señal) 0.66 que con $p$ (señal) 0.33 . Durante las demoras no señaladas la tasa de respuesta se mantuvo constante en función de la duración de la demora y del valor de p(señal) en combinación con ambos intervalos entre reforzadores. Los resultados sugieren que la tasa de 
respuesta durante los periodos de demora estuvo determinada principalmente por la tasa durante las demoras señaladas. Complementariamente, las demoras obtenidas fueron más cortas cuando se señaló la demora con el intervalo entre reforzadores de $128 \mathrm{~s}$ que con el intervalo de $32 \mathrm{~s}$. En el caso de las demoras no señaladas, las demoras obtenidas fueron similares para los dos intervalos entre reforzadores. Las diferencias en la tasa de respuesta y en las demoras obtenidas fueron más acentuadas con $p$ (señal) 0.66 que con $p$ (señal) 0.33 . Estos resultados sugieren que la transición de un efecto de frecuencia de reforzamiento a uno de tiempo relativo al incrementar la probabilidad de presentar una señal durante el periodo de demora, lejos de ser gradual se debe a un efecto de todo o nada, donde presentar una señal o no presentarla determina un efecto $u$ otro. El aparente efecto gradual de incrementar el valor de $p$ (señal) sobre la tasa de respuesta global podría deberse a promediar la tasa de respuesta cuando se presenta la señal y cuando no se presenta, de tal manera que conforme mayor es la proporción de demoras señaladas se obtiene un efecto cada vez más cercano a un claro efecto de tiempo relativo.

El hecho de que presentar o no presentar una señal durante el periodo de demora determina la ocurrencia de un efecto de frecuencia de reforzamiento o uno de tiempo relativo es consistente con la literatura sobre reforzamiento condicionado. Esto es, presentar una señal precedente al reforzador resulta en tasas de respuesta más altas que cuando no se presenta ninguna señal. Los resultados del presente estudio apoyan la sugerencia de Spence (1947, 1956), de que un procedimiento de demora de reforzamiento señalada implica una demora en la entrega del reforzador pero al mismo tiempo la presentación inmediata de un estímulo que adquiere la función de reforzador condicionado, lo cual mantiene una tasa de respuesta más alta que cuando solo existe una demora en la entrega del reforzador (veáse Grice, 1948; Lawrence \& Hommel, 1961 y Perkins, 1947 como evidencia empírica del argumento de Spence). El aumento observado en la tasa de respuesta durante el periodo previo a la demora (IA) y durante la demora conforme aumentó el valor de $\mathrm{p}$ (señal), probablemente se debió a que la señal durante la demora adquirió una función de reforzador condicionado para las respuestas durante los periodos precedentes a la señal (IA) y de estímulo discriminativo para las respuestas que ocurrieron durante los periodos de demora, conforme aumentó la frecuencia de apareamientos señal-reforzador (cf., Bersh, 1951; Dinsmoor, 1950; Hall, 1951; Miles, 1956; véase Myers, 1958, como una revisión del papel de una señal como estímulo discriminativo y como reforzador condicionado). En otros estudios en los que se ha variado la probabilidad de presentar una señal precedente al reforzador se ha mostrado que manipular la probabilidad de presentar un estímulo que antecede a la presentación del reforzador controla el nivel de la tasa de respuesta, conforme mayor es la probabilidad de presentar el estímulo mayor es la tasa de respuesta (e.g., 
Bruner, 1982, en automoldeamiento; Nevin, 1964, en programas de reforzamiento encadenados).

\section{REFERENCIAS}

Avila, R. \& Bruner, C. A. (1989). Efectos del intervalo respuesta-reforzador y del ciclo de reforzamiento en un programa de demora variable. Revista Mexicana de Análisis de la Conducta, 15, 23-39.

Azzi, R., Fix, D. S. R., Keller, F. S., \& Rocha e Silva, M. I. (1964). Exteroceptive control of response under delayed reinforcement. Journal of the Experimental Analysis of Behavior, 7, 159-162.

Bersh, P. J. (1951). The influence of two variables upon the establishment of a secondary reinforcer for operant responses. Journal of Experimental Psychology, 41, 62-73.

Bruner, C. A. (1982). El efecto de variar la probabilidad del estímulo en "automoldeamiento/automantenimiento". Revista Mexicana de Análisis de la Conducta, 8, 47-56.

Bruner, C. A., Avila, R., Acuña, L. \& Gallardo, L. M. (1998). Effects of reinforcement rate and delay on the acquisition of lever pressing by rats. Journal of the Experimental Analysis of Behavior, 69, 59-75.

Daniel, W. W. (1978). Applied Nonparametric Statistics. Boston, MA: Houghton Mifflin Company.

Deese, J. \& Hulse, S. H. (1967). The Psychology of Learning. 3a. Edición. Tokyo: McGraw-Hill Kogakusha, Ltd.

Dinsmoor, J. A. (1950). A quantitative comparison of the discriminative and reinforcing functions of a stimulus. Journal of Experimental Psychology, 40, 458-472.

Fantino, E. (1969). Choice and rate of reinforcement. Journal of the Experimental Analysis of Behavior, 17, 3-14.

Grice, G. R. (1948). The relation of secondary reinforcement to delayed reward in visual discrimination learning. Journal of Experimental Psychology, 38, 1-16.

Hall, J. F. (1951). Studies in secondary reinforcement: I. Secondary reinforcement as a function of the frequency of primary reinforcement. Journal of Comparative and Physiological Psychology, 44, 246-251.

Kimble, G. A. (1961). Hilgard and Marquis' Conditioning and Learning. New York: Appleton-century-Crofts.

Lattal, K. A. (1984). Signal functions in delayed reinforcement. Journal of the Experimental Analysis of Behavior, 42, 239-253.

Lattal, K. A. (1987). Considerations in the experimental analysis of reinforcement delay. En: M. L. Commons, J. E. Mazur, J. A. Nevin, \& H. Rachlin (Eds.), Quantitative Analyses of Behavior: Vol. 5. The Effect of Delay and of Intervening Events on Reinforcement Value (pp. 107-123). Hillsdale, NJ: Erlbaum.

Lawrence, D. \& Hommel, L. (1961). The influence of differential goal boxes on discrimination learning involving delay of reinforcement. Journal of Comparative and Physiological Psychology, 54, 552-555. 
Miles, R. C. (1956). The relative effectiveness of secondary reinforcers throughout deprivation and habit-strength parameters. Journal of Comparative and Physiological Psychology, 49, 126-130.

Myers, J. L. (1958). Secondary reinforcement: A review of recent experimentation. Psychological Bulletin, 55, 284-301.

Nakajima, S. (1994). Contextual control of Pavlovian bi-directional occasion setting. Behavioral Processes, 32, 53-66.

Nevin, J. A. (1964). Two parameters of conditioned reinforcement in a chaining situation. Journal of Comparative and Physiological Psychology, 58, 367-373.

Perkins, C. C. (1947). The relation of secondary reward to gradients of reinforcement. Journal of Experimental Psychology, 37, 377-392.

Reilly, M. P. \& Lattal, K. A. (2004). Within- session delay-of-reinforcement gradients. Journal of the Experimental Analysis of Behavior, 82, 21-35.

Renner, K. E. (1964). Delay of reinforcement: A historical review. Psychological Bulletin, 61, 341-361.

Richards, R. W. (1981). A comparison of signaled and unsignaled delay of reinforcement. Journal of the Experimental Analysis of Behavior, 35, 145-152.

Schaal, D. W. \& Branch, M. N. (1988). Responding of pigeons under variable-interval schedules of unsignaled, briefly signaled, and completely signaled delays to reinforcement. Journal of the Experimental Analysis of Behavior, 50, 33-54.

Schaal, D. W., Odum, A. L., \& Shahan, T. A. (2000). Pigeons may not remember the stimuli that reinforced their recent behavior. Journal of the Experimental Analysis of Behavior, 73, 125-139.

Schaal, D. W., Schuh, K. J. \& Branch, M. N. (1992). Key pecking of pigeons under variable-interval schedules of briefly signaled delayed reinforcement: effects of variableinterval value. Journal of the Experimental Analysis of Behavior, 58, 277-286.

Schneider, S. M. (1990). The role of contiguity in free-operant unsignaled delay of positive reinforcement: A brief review. The Psychological Record, 40, 239-257.

Shahan, T. A. \& Lattal, K. A. (2005). Unsignaled delay of reinforcement, relative time, and resistance to change. Journal of the Experimental Analysis of Behavior, 83, 201-219.

Sizemore, O. J. \& Lattal, K. A. (1978). Unsignaled delay of reinforcement in variableinterval schedules. Journal of the Experimental Analysis of Behavior, 30, 169175.

Spence, K. W. (1947). The role of secondary reinforcement in delayed reward learning. Psychological Review, 54, 1-8.

Spence, K. W. (1956). Behavior Theory and Conditioning. New Haven, CO: Yale University Press.

Tarpy, R. M. \& Sawabini, F. L. (1974). Reinforcement delay: A selective review of the last decade. Psychological Bulletin, 81, 984-997.

Terrace, H. S. (1966). Stimulus control. En: W. K. Honig (Ed.), Operant Conditioning: Areas of Research and Application (pp. 271-344). Appleton-Century-Crofts.

Williams, B. A. (1998). Relative time and delay of reinforcement. Learning and Motivation, 29, 236-248.

Williams, B. A. (2003). Challenges to timing-based theories of operant behavior. Behavioral Processes, 62, 115-123. 